\title{
Open Source Intelligence's Methodology Applied to Organizational Communication
}

\author{
Stefania Fantinelli \\ Domenico Franco Sivilli \\ Università degli Studi G. d'Annunzio Chieti Pescara \\ Email: stefania.fantinelli@unich.it, fsivilli@unich.it
}

Doi:10.5901/mjss.2015.v6n2p233

\section{Abstract}

Every minute in the world tons of information is generated online through the net. Every single bit could represent a source of potential knowledge for public and private organizations. The purpose of this study is to illustrate how implementing Open Source Intelligence (OSINT) methodology within organizational management can strengthen the brand reputation and competitive intelligence activities. OSINT technique could be also applied to the psychosocial research in order to extend its boundaries, for example in the field of sentiment analysis, opinion tracking and user profiling. The OSINT methodology has been analysed by the psychological organizational theories: Burns and Stalker (1971) claimed that the organization is an organic system strictly connected with the external dynamic context. Later on, Butera (1992) defined the organization as an organism concerned with both internal and external communication, therefore open source intelligence can facilitate a right management of information and knowledge. OSINT represents a new and wide-scoped instrument to gather data and information for the organizations to improve decision-making, conduct preventive risk analyses, enhance the due-diligence information acquisition processes, monitor the effectiveness of organizational communication and online reputation. Open source intelligence is already deeply linked with social sciences and should be part of enterprises' organizational activity. What are the challenges related to implementing a successful OSINT strategy in the organization's communication model?

Keywords: organization, communication, OSINT, brand reputation, competitive intelligence.

\section{Introduzione}

I mass-media costituiscono i principali mezzi di informazione e sono anche rappresentativi di opinioni ed interessi delle persone; secondo la field theory di Kurt Lewin (1951) il comportamento di ogni singolo individuo dipenderà dal contesto $\mathrm{e}$ dalle interazioni con l'ambiente, influenzate dalle proprie caratteristiche di personalità. Lewin focalizza l'attenzione sui real-world issues, fenomeni concretamente rilevanti, che potrebbero essere veicolati dalla comunicazione informativa. Ritroviamo questa dinamica anche nella comunicazione organizzativa: quando le informazioni sono rivolte ad un vasto pubblico il processo di influenza sarà duplice, dal momento che le singole persone in un gruppo si condizionano reciprocamente.

Stravolgimenti comunicativi hanno interessato anche l'universo commerciale ed organizzativo, che si è concentrato in particolare sulle possibilità offerte da Internet: il web rappresenta infatti un potente mezzo per curare il proprio brand, fare pubblicità, influenzare gli atteggiamenti dei consumatori ed, essendo una fonte aperta, è allo stesso tempo un'inesauribile risorsa per raccogliere opinioni e monitorare i comportamenti delle persone.

Una delle tecniche maggiormente in uso per effettuare intelligence da fonti aperte è l'analisi semantica, che può essere definita come l'insieme di analisi grammaticale, logica e sintattica di una frase, operando contestualmente il riconoscimento di concetti e contenuti. La notevole mole di informazioni a disposizione degli analisti non facilita il loro compito ma al contrario rappresenta il maggiore ostacolo, per questa ragione è indispensabile dare la giusta rilevanza al progetto ed al controllo durante l'analisi (ExpertSystem.it 2011). Al fine di supportare tali attività sono stati elaborati programmi per la comprensione ed analisi delle informazioni. II principale strumento tecnologico utilizzato per l'Open Source Intelligence è il metodo del Text Mining, che consente di effettuare in modo automatico analisi, comprensione dei testi, categorizzazione ed indicizzazione di argomenti e concetti espressi (Nacci 2008). Si tratta infatti di un'analisi linguistica e statistica: linguistica in quanto consente di ottenere informazioni morfo-sintattiche, logiche e semantiche. L'analisi statistica guida le azioni di clustering e categorizzazione. I software sviluppati per applicare tale metodo sono diversi e consentono di isolare ed analizzare tutti i tipi di informazioni provenienti da una abituale comunicazione effettuata via mail, sms, su una pagina web o ancora tramite articoli. Viene riprodotto un processo simile a quello 
effettuato dalle persone nella comprensione di una comunicazione interpersonale, tanto che questi software hanno anche capacità di disambiguazione: alcune parole, sia in italiano che in inglese, possono avere diversi significati pur avendo la stessa forma. Nell'avviare una ricerca è possibile determinare a priori il significato specifico a cui fare riferimento; inoltre nel momento dell'analisi, se si incontra una parola che può riferirsi a più concetti, il significato verrà contestualizzato, quindi attribuito in base al senso complessivo della frase espressa.

II vantaggio competitivo per un'organizzazione è rappresentato dalla tempestività di tale metodologia: un'analisi semantica del contenuto può essere applicata anche in tempo reale ai contenuti ed alle sensation emergenti dai social network e possono essere messe in evidenza le argomentazioni, anche in ordine di frequenza (ExpertSystem.it 2011). Approfondire la conoscenza delle percezioni, dei sentimenti e delle aspettative degli utenti rappresenta un valido strumento per le aziende per poter monitorare la reputazione del proprio brand o dei propri prodotti. Come esito del processo può essere creata una mappa concettuale a partire dagli utenti e dalle loro relazioni o dagli argomenti espressi.

L'analisi delle fonti aperte è un'attività che prevede un approccio interdisciplinare; racchiude in sé elementi di logica, linguistica e scienze cognitive, chiamando in causa, in particolare, psicologia e sociologia. L'analista apporta la propria conoscenza ed esperienza per estrarre ed evidenziare i contenuti rilevanti.

I primi accostamenti dell'intelligence alle scienze sociali ed alla psicologia in particolare si hanno nel Novecento con l'affermarsi dei primi fondamenti di psicologia moderna. I lavori di Brentano (1874) e Wundt (1874) determinano la nascita della psicologia come disciplina scientifica; lo sviluppo successivo di teorie in ambito psicosociale seguirà di pari passo la crescita dei servizi segreti (Giannuli 2009). Le ricerche sulla psicologia delle folle (Le Bon 1895), sull'opinione pubblica (Bernays 1928), sui riflessi condizionati (Pavlov 1903) e sul controllo operante (Skinner 1948), verranno utilizzate come supporto e fondamento psicologico per attività spesso immorali ed in contrasto con le originali intenzioni degli autori, quali ad esempio: la disinformazione, l'inganno, la propaganda, la guerra psicologica.

II paradigma di analisi delle informazioni disponibili tramite le fonti aperte è nel contempo diventato uno strumento di vantaggio nei settori sociale, organizzativo e per la ricerca psicosociale; ad esempio, la rilevazione di atteggiamenti ed opinioni può essere effettuata nella comunità virtuale, applicando una sorta di osservazione naturalistica, oppure la cosiddetta netnografia (Kozinets 2000) si può accostare in triangolazione ad altre modalità di ricerca, quali ad esempio focus group 0 interviste. L'indagine sul mood o sul sentiment della nazione può essere effettuata tramite tecniche di analisi semantica del contenuto, il cosiddetto tracking delle opinioni potrà interessare social network, blog, forum, website tradizionali.

\section{II Valore della Comunicazione nel Contesto Organizzativo}

Reciprocità, scambio, apertura, condivisione, sono elementi imprescindibili di qualsiasi attività comunicativa e caratterizzano anche la comunicazione organizzativa. In queste attività sono coinvolti i dipendenti, i clienti acquisiti e potenziali, stakeholder interessati o comunque implicati nelle attività dell'organizzazione; per tale ragione si può parlare di comunicazione integrata, che comprende, in un'ottica sinergica, ambiente interno ed esterno (Invernizzi 1993). Con gli studi di Putnam et al. (1996) la comunicazione diviene linfa vitale di ogni tipo di organizzazione; non è più intesa come uno strumento bensì come fulcro attorno al quale si pianifica il lavoro dell'organizzazione considerando sempre interno ed esterno contemporaneamente.

Nel modello delle "4 C" (cooperazione, comunicazione, conoscenza, comunità) elaborato da Federico Butera (1999) è sottolineata l'importanza del legame con le fonti di conoscenza provenienti anche dall'esterno: comunicazione e conoscenza estese e condivise sono tra le attività necessarie e indispensabili per un'organizzazione nell'era dell'informazione. Diviene naturale un rapporto continuo, reciproco ed osmotico tra ambiente interno ed esterno (Romano e Felicioli 1992) che può essere supportato e intensificato dalle nuove tecnologie.

La comunicazione integrata influenza e produce effetti diretti su quattro ambiti della vita organizzativa: l'immagine, la reputazione, il clima organizzativo interno e la cultura organizzativa. Come osserva Crozier (1990) è opportuno che l'organizzazione sia predisposta all'ascolto per poter osservare i comportamenti degli stakeholder esterni, essere presente in modo attivo sul mercato e comunicare con i consumatori, adattandosi in questo modo ai cambiamenti che si verificano nell'ambiente esterno ed alle relative richieste (Drucker 1993), di conseguenza il pubblico percepisce di essere parte attiva di un circolo comunicativo e sviluppa maggior committment.

Quasi in concomitanza con il massiccio sviluppo dell'Information Technology sono state condotte numerose ricerche con l'intento di verificare che tipo di impatto potessero avere le nuove tecnologie nel contesto organizzativo. I risultati mostrano che i maggiori cambiamenti si sarebbero verificati in particolare sotto quattro punti di vista differenti:

- Miglioramento del sistema di comunicazione e dell'accesso alle informazioni per ottimizzare e supportare il processo di decision making. In un momento in cui si registra un notevole incremento di informazioni 
provenienti da fonti differenti, che richiedono un'analisi complessa propedeutica alle future decisioni, diventano del tutto sconvenienti e penalizzanti i metodi tradizionali di analisi manuale delle informazioni. Per quanto riguarda l'intervento sulla comunicazione, nasce nei primi anni ' 90 un sistema di supporto al lavoro di gruppo, per facilitarne le interazioni; si tratta essenzialmente di implementazione di strumenti come la posta elettronica e la videoconferenza (Leidner and Elam, 1995);

- La competizione tra organizzazioni sfrutta il supporto delle nuove tecnologie per ottenere maggiori vantaggi; acquisisce maggior rilievo l'ascolto del cliente, ad esempio si apportano modifiche ai propri prodotti in risposta ai pareri del pubblico (Clemons and Row, 1991);

- Nascita di nuovi modelli di business: c'è la possibilità di migliorare il rapporto con la clientela, anche solo offrendo un servizio di customer service continuo. Per la prima volta le organizzazioni hanno l'opportunità di presentare online, sul web, i propri servizi o prodotti potendo espandere in modo esponenziale il proprio commercio (Drucker, 1988);

- L'information technology offre supporto alle attività normalmente svolte dall'uomo, un aumento dell'efficienza e la diminuzione dei costi possono condurre ad un ampliamento del business (Tapscott and Caston, 1993).

In particolare negli ambiti della comunicazione e del decision making si registrano tuttora incessanti sviluppi e ci saranno ancora notevoli cambiamenti scaturiti da nuovi utilizzi dell'information technology.

\section{Le Fonti Aperte: Fonti di Conoscenza e Potere}

"Fonti aperte" sono tutti quei dati accessibili da un punto di vista giuridico a chiunque. II termine fonte si riferisce a qualsiasi entità portatrice di un certo contenuto informativo: qualsiasi ente 0 istituzione, individui singoli 0 gruppi, enti di formazione, associazioni, database, giornalismo di diverso genere, Internet e quindi forum, blog, siti, e-mail, chat, etc. Dal momento che viviamo nella società dell'informazione abbiamo a disposizione innumerevoli mezzi da cui poter ricavare notizie. Si crea una sovrapposizione tra mezzo di comunicazione e messaggio trasmesso; per comprendere meglio e completare la definizione di fonte possiamo prendere in prestito alcuni concetti dalle teorie di Goffman (1981): una fonte, come l'emittente di una comunicazione, racchiude in sé i tre ruoli di animatore, autore e mandante. Queste funzioni possono coesistere nella fonte 0 essere distribuite tra entità differenti, individui, organizzazioni o gruppi.

"Aperta" non significa che debba essere necessariamente gratuita, ma indica un'informazione nata originariamente per essere comunicata o diffusa; possiamo distinguere la risorsa aperta da quella classificata che è creata in origine per una distribuzione limitata e ad accesso regolamentato (Nacci 2007).

Applicando il concetto di intelligence alle fonti aperte si materializza nell'immaginario comune la figura della spia, ma in realtà occorre specificare che vi sono differenze tra analista di fonti aperte e l'agente segreto dei romanzi. Partendo dall'origine dei due termini, dalla loro etimologia, l'analista è colui che scompone, che risolve, che scioglie; ci si riferisce all'approccio di scomposizione del tutto in elementi più semplici. La spia è colui che esplora e che riferisce, operando quindi un passaggio dall'astratto al concreto delle informazioni in origine riservate. Lo spionaggio è inteso come la pratica di ottenere segreti, mentre nel caso dell'analista di fonti aperte non è corretto parlare di segreti; le informazioni trattate possono essere più o meno accessibili, ma mai definibili segreti. L'Analista di Intelligence è colui che, al servizio di enti ed istituzioni pubbliche o private, trasforma la semplice informazione in intelligence e la rende comprensibile ed utilizzabile per il decisore finale; le informazioni hanno ovviamente diverso valore in base all'ambito di provenienza o di applicazione finale (Sidoti 2000).

II primo documento scritto che sostiene ed analizza l'importanza della raccolta delle informazioni è il trattato di strategia ed arte militare attribuito a Sun Tzu, risalente al III secolo a.C. II testo è particolarmente illuminante ed ancora attuale nei contenuti, tanto che non solo generali ma anche manager e decisori politici lo utilizzano come guida nella gestione delle loro attività. Ecco come l'analisi delle fonti aperte può avere utili risvolti anche per le organizzazioni, che possono sfruttare strumenti basati su algoritmi di analisi semantica per identificare, ad esempio, trend di gradimento di un prodotto appena immesso sul mercato, per monitorare i competitor, individuare strategie d'azione. L'utilizzo delle fonti aperte interessa diversi settori: privato, economico organizzativo, istituzionale; si parla infatti di security aziendale, business intelligence ed intelligence governativa ma anche delle pratiche di due diligence. II fine ultimo sarà diverso a seconda che si tratti di ambito istituzionale, privato o aziendale: tra questi campi di applicazione la differenza sostanziale si trova nel contenuto e nell'origine delle fonti e non nel processo di analisi che è ugualmente caratterizzato da quattro fasi (Caligiuri 2002):

- Scoperta: sapere dove cercare è l'elemento centrale dell'OSINT, o come sostengono gli esperti del settore è necessario sapere chi detiene l'informazione; quindi "conoscere chi conosce"; 
- distinzione: è fondamentale poi fare un'attenta distinzione tra fonti rilevanti ed irrilevanti, aggiornate o meno e tra fonti con un buon rapporto tra costi e benefici e fonti con costi eccessivi;

- distillazione: è il passaggio più importante in cui viene effettuata l'analisi ed il sunto, quindi bisogna inserire le informazioni rilevanti in uno spazio contenuto in modo efficace;

- distribuzione: il prodotto del processo di intelligence deve giungere in tempi brevi ed in una forma comprensibile e compatibile all'utilizzatore.

In particolare nelle fasi centrali si opera il passaggio da dato grezzo ad informazione rilevante che andrà a dare vita al circolo virtuoso tra informazione, conoscenza e potere (Foucault 1984); quest'ultimo da intendersi come disponibilità all'azione, facoltà di prendere decisioni guidate dalla conoscenza e definire modelli di business in ambito organizzativo o interventi politico-strategici in ambito governativo.

\section{Brand Reputation: Dalle Fonti Aperte alla Comunicazione Digitale}

Per brand reputation si intende l'attività di gestione e costruzione della reputazione aziendale o di uno specifico marchio 0 prodotto; essa rappresenta un vero e proprio investimento e, se curata efficacemente, consente di gestire l'identità aziendale dall'interno e dall'esterno, prestando attenzione agli stakeholder ed alle loro opinioni e commenti, aumentando la conoscenza e l'accettazione del marchio nel pubblico ed influenzando indirettamente quindi il fatturato. È necessario specificare la differenza tra immagine e reputazione: la prima fa riferimento all'impressione 0 all'idea che una persona 0 una azienda fornisce di sé al pubblico in base all'aspetto 0 al modo in cui si presenta. L'immagine è creata nel presente, è effimera e la sua creazione non coinvolge attivamente i dipendenti né un'azione comunicativa, tanto che online l'immagine è rappresentata dal sito istituzionale, illustrativo dei prodotti.

La reputazione riguarda invece qualcosa di più profondo e radicato: la stima o la considerazione in cui si è tenuti da altri; dura a lungo, si costruisce nel corso del tempo, chiama in causa azioni e comportamenti. In tal caso la comunicazione ha un ruolo primario, anche i dipendenti sono motivati a seguire un determinato codice di comportamento che rispecchi l'identità ed i valori aziendali: non bisogna dimenticare infatti che i primi stakeholder influenti sono i dipendenti. Già nel 2000 nel Cluetrain Manifesto le imprese venivano esortate ad "occuparsi della reputazione e non dell'immagine!".

L'Open Source Intelligence rappresenta uno dei modi per gestire la propria brand reputation: curare la propria presenza online, controllare i competitor e monitorare le opinioni espresse dai clienti/utenti. Con l'avvento di Internet e del web 2.0 controllare il proprio status diventa sempre più importante, e nasce anche il fenomeno definito ego-surfing (Ivone 2005): è spesso il manager in prima persona ad effettuare delle ricerche sul web per trovare tracce della propria presenza. Anche questo è un modo per curare la brand reputation di un'azienda e per migliorare i propri standard: infatti nel momento in cui un manager dovesse venire a conoscenza di uno scontento 0 di alcune critiche rivolte ai prodotti 0 ai servizi della propria azienda, potrà mettere in atto gli opportuni cambiamenti, intervenendo negli interessi dell'organizzazione.

Allo scopo di curare la propria reputazione spesso le organizzazioni creano dei blog aziendali per favorire la comunicazione e soprattutto per tentare un bilanciamento comunicativo: normalmente infatti esiste uno squilibrio tra l'organizzazione da un lato, e gli stakeholder dall'altro, che recepiscono ed interpretano i messaggi in base ai quali poi regoleranno i propri comportamenti di consumo. Se analizziamo il contesto organizzativo alla luce di una "pragmatica della comunicazione organizzativa" (Cortini 2005), risulta di fondamentale importanza l'interattività del web 2.0 che consente uno scambio reciproco immediato; dunque gli assiomi teorizzati da Watzlawick (1971) si possono ben applicare a questo contesto. Ad esempio con il primo assioma - è impossibile non comunicare - si intende sottolineare l'importanza della comunicazione verbale e non verbale tra individui: ogni singola azione acquista un significato. Traslando questo concetto nel mondo organizzativo il senso non varia: ogni comportamento e comunicazione verbale, scritta, per immagini o simboli verrà percepita ed interpretata dai molteplici riceventi.

Il blog creato dall'azienda rappresenta un mezzo di comunicazione accessibile anche dai dipendenti, ma bisogna considerare alcune criticità: potrebbe essere minacciato il delicato equilibrio tra gli interessi dell'azienda ed il diritto di libera espressione dei dipendenti. Uno degli obiettivi di un employee blog consiste nel comunicare l'immagine dell'azienda, oltre che facilitare la comunicazione interna ed esterna, ma le esperienze lavorative riportate dal blogger 0 commenti personali sull'organizzazione potrebbero trovarsi in contraddizione con i valori dell'azienda stessa.

È interesse dell'organizzazione supervisionare costantemente le conversazioni pubblicate sui blog e sugli strumenti ufficiali (Cortini 2008) al fine di ridurre i rischi derivanti dall'esposizione di informazioni sensibili o inappropriate; nel 2002 si parla per la prima volta di doocing, indicando con tale termine il licenziamento di un dipendente dovuto alla pubblicazione di contenuti non conformi alla cultura aziendale (Cortini 2009). Come già accennato questo fenomeno 
chiama in causa aspetti etici e legali nonchè il corretto utilizzo delle tecnologie al servizio dell'organizzazione; diventa indispensabile una formazione mirata all'implementazione degli strumenti e all'adozione di comportamenti consoni al media, l'estensione della netiquette anche all'utilizzo degli strumenti aziendali, blog incluso.

Attualmente grazie ai social network il trasferimento di informazioni, le interazioni e la condivisione di contenuti avvengono sotto gli occhi di tutti. Da un lato l'organizzazione funge da entità sorvegliante e limitante per i propri dipendenti; ma dall'altro dinamicità ed immediatezza permettono di comprendere in tempo reale le richieste e mettersi in diretto contatto con il pubblico, oltre che applicare anche il più classico dei principi del marketing: non vendere un prodotto, ma creare un bisogno nel destinatario.

\section{Competitive Intelligence e Confronto Organizzativo}

La competitive intelligence è un'insieme di azioni e strategie etiche, legali e sistematiche messo in atto da aziende per avere informazioni sulle attività, intenzioni, capacità e vulnerabilità della concorrenza nonchè sulle attuali tendenze $e$ sviluppi del mercato, e prendere infine le decisioni conseguenti (Miller 2001).

Secondo Herring (1996), esperto di intelligence, i possibili ambiti di applicazione di tale attività sono: prevenire sorprese all'organizzazione di appartenenza fornendo servizi di early warning (avvisi che si riferiscono ai primi segnali provenienti dalla concorrenza o dal mercato); supportare il processo decisionale con adeguate informazioni; individuare e mantenere sotto controllo i competitor; contribuire a sviluppare strategie di mercato e comunicative; svolgere infine un ruolo chiave nella raccolta e nel reporting delle informazioni (Malfanti 2005). L'azienda che applica strategie di competitive intelligence sarà in grado di anticipare i movimenti del mercato, anziché reagire ad essi; atteggiamento fondamentale in un corretto apparato di crisis management dove non è importante solo saper reagire ad una crisi sia interna che esterna, ma essere soprattutto in grado di anticiparla.

È inoltre possibile applicare questo strumento in tutti i settori di un'azienda ed in tutti gli ambiti decisionali, dal tattico allo strategico (Bonacina 2005). Allo scopo di mettere a fuoco cambiamenti in atto, tendenze sul mercato, pericoli o vulnerabilità, vengono raccolte informazioni che poi, correlate tra loro, daranno luogo ad una conoscenza strutturata. II processo seguito è lo stesso dell'attività di intelligence vera e propria. Si svolge in modo continuo attraverso fasi cicliche: innanzitutto bisogna pianificare l'obiettivo dell'analisi e quindi comprendere le necessità espresse dal decisore finale, iniziando successivamente le attività di raccolta dati ed analisi degli stessi per trasformarli in informazioni fruibili che verranno poi distribuite agli utilizzatori finali (Herring 1998). Questi ultimi forniranno un feedback come espressione di nuovi bisogni, da cui prenderà inizio il successivo ciclo di attività. Dunque con l'utilizzo di strumenti pienamente legali si giunge alla scoperta, sviluppo e diffusione di una conoscenza che sarà di supporto all'attività decisionale.

Conoscendo le attività, gli elementi distintivi e le debolezze dei propri competitor, potrebbe verificarsi lo stesso processo che avviene nelle interazioni sociali: Festinger (1954) lo chiamò "confronto sociale" parlando di individui, in questa sede possiamo definirlo "confronto organizzativo".

Nei rapporti interpersonali, soprattutto in momenti di incertezza, un individuo spesso ricorre al confronto con gli altri per completare l'immagine che ha di sé e per curare la propria identità. L'esito del confronto ha importanti effetti sul mantenimento della propria autostima. Immaginiamo come tali azioni comparative possano essere effettuate anche da organizzazioni ed il confronto, come teorizzato da Festinger, possa avvenire su tre livelli differenti con obiettivi ed esiti distinti:

- un confronto verso l'alto può essere stimolante e rappresentare una spinta al miglioramento;

- verso il basso è un confronto privo di stimolazioni ma in grado di innalzare i livelli di autostima;

- il confronto con i simili è quello più realistico, grazie al quale si possono trarre valutazioni sul proprio operato.

Se nelle relazioni sociali la percezione del livello relativo del termine di paragone scelto può essere del tutto soggettiva, per le organizzazioni tale percezione sarà dettata da riscontri numerici e dimensionali, e quindi più oggettiva e realistica.

Ipotizziamo che il confronto possa avere implicazioni sul clima organizzativo, che è condizione interna ma strettamente connesso alle impressioni e percezioni provenienti dall'ambiente esterno (Quaglino e Mander 1987).

\section{Conclusioni}

Nell'era dell'informazione il lavoro di intelligence, che abbia finalità civili, militari, di ricerca accademica e sociale 0 di brand reputation, non può prescindere dall'utilizzare per le proprie analisi le fonti aperte. L'enorme mole di informazioni in continua e rapida crescita definisce in parte il concetto di Big Data e rappresenta una delle frontiere dell'attuale tecnologia: elaborare dati fornendo risposte pertinenti in tempi utili, divenendo così la potenziale ricchezza del nuovo 
millennio.

La rilevazione del sentiment dei clienti nei confronti di un marchio o di un prodotto e la ricerca sulle relazioni sociali e gli interessi degli utenti e dei gruppi sono solo alcuni dei campi d'applicazione per i quali l'OSINT rappresenta un vantaggio competitivo, se non un vero e proprio requisito.

Per implementare questi servizi un'organizzazione può rivolgersi ad una società di consulenza esterna e mettere a punto strategie di brand reputation o social media analysis per monitorare il mercato proprio e dei competitor. Una diversa punteggiatura degli eventi (Watzlawich 1971) o le possibili incomprensioni tra identità professionali differenti, rappresentano le maggiori criticità in questo scenario. È fondamentale per l'organizzazione avere obiettivi molto chiari ed essere in grado di comunicarli. Spesso però, come accade nel mondo dell'intelligence governativa, l'approccio mentale dei partecipanti è diverso: esiste un divario tra i metodi dell'analista e quelli del decisore politico o utilizzatore finale delle informazioni. Solo per fare alcuni esempi: l'analista di intelligence ha come priorità la perfezione e completezza dell'informazione fornita, mentre l'utilizzatore finale ha come obiettivo l'urgenza di ottenere informazioni sufficientemente valide, utili e attendibili per consentire un'azione decisionale; inoltre la visione a lungo termine dell'analista si contrappone a quella contingente del decisore (Steele 2002).

Differentemente un'organizzazione può investire al proprio interno, dedicare una posizione al ruolo di social media analyst e sarà dunque necessario progettare un intervento formativo ad hoc. Come proposto da Salas et al. (1999) gli obiettivi della formazione interessano sempre tre dimensioni, riassunte nell'acronimo KSA: knowledge, skill, ability or attitude. Nel nostro caso sarà sicuramente una formazione interdisciplinare che spazierà tra marketing, informatica, giornalismo, scienze sociali e psicologia.

Non è facile delineare la figura del formatore per un'attività di competitive intelligence e brand reputation, se pensiamo che nel 2006 si discuteva ancora sull'identità della competitive intelligence: se fosse da inquadrare come professione, come disciplina o come settore commerciale (Wagner 2006). Fino alla pubblicazione del Body of Knowledge (BOK) avvenuta nel 2009 a cura dello SCIP (Society of Competitive Intelligence Professionals), i professionisti che operavano nel settore non potevano usufruire di metodologie validate, né era stato attivato alcun percorso formativo specifico (Shelfer 2003, McGonagle 2003). II BOK descrive 86 competenze che riguardano la gestione e la realizzazione concreta dell'attività; non vengono definite nel dettaglio le soft skill richieste proprio per la varietà di applicazioni della competitive intelligence. Dunque le attitudini, le capacità critiche, le abilità comunicative e di interazione si dovranno adattare di volta in volta al contesto ed agli obiettivi dell'intervento. Michaeli (2010) suggerisce alcune caratteristiche che possano rendere efficiente un intervento formativo: lezioni individuali piuttosto che collettive, discussioni e attuazione di progetti in team, esercitazioni sul campo e attività di mentoring. Le metodologie di open source intelligence possono essere utilizzate dall'organizzazione anche per gestire le proprie conoscenze interne, rinforzando l'esistente ciclo conoscenza-potere come ipotizzato da Foucault (1984).

Diventa indispensabile in organizzazioni di ogni dimensione e ambito sviluppare interventi formativi dedicati a tutti i dipendenti relativamente all'uso di social media, entrati ormai nella sfera quotidiana personale e lavorativa, ricordando che sono proprio i dipendenti i primi rappresentanti dell'organizzazione, portatori di valori e dell'identità aziendale all'esterno.

Ci si può domandare se all'interno di una organizzazione tutti i dipendenti abbiano lo stesso accesso alle tecnologie, facendo riferimento ad una sorta di digital divide organizzativo (Stephens e Dailey 2014), dovuto ad esempio a competenze informatiche e digitali di diverso livello, ed una diversa capacità e competenza nell'utilizzo degli stessi, che comporterà l'applicazione di approcci disomogenei agli strumenti informatici e di comunicazione. In tali casi potrebbe bastare l'erogazione della formazione tecnologica, o bisognerebbe educare ad una nuova cultura della comunicazione digitale?

L'obiettivo del presente lavoro è essenzialmente descrittivo e vuole porre l'attenzione su come i metodi di open source intelligence possano espandere il loro bacino di interesse, occupando aree nel mondo del marketing, del management e della ricerca psico-sociale.

Prendendo spunto dai concetti espressi ipotizziamo una futura linea di ricerca che preveda una triangolazione combinando metodi qualitativi e quantitativi: un questionario dedicato ad organizzazioni ed analisti, focus group per i responsabili della comunicazione nelle organizzazioni e interviste delphi per gli analisti di fonti aperte. In questo modo sarà possibile approfondire la situzione dal punto di vista di professionisti del settore, identificare le attività praticate $\mathrm{e}$ quali siano i principali vantaggi e le maggiori criticità. Si potrà verificare se l'implementazione di metodologie OSINT all'interno di organizzazioni possa migliorare o semplicemente supportare le attività di brand reputation grazie all'aumentata comprensione di informazioni disponibili ed infine pianificare interventi formativi e capire quali sono le best practice o le migliori strategie applicate. 


\section{Riferimenti Bibliografici}

Bernays, E.L. (1928). Propaganda, New York Liveright.

Bonacina, S. (2005). "La Competitive Intelligence per la azienda di oggi e domani", in Business Online, 9 gennaio.

Brentano, F. (1874). Psychologie vom empirischen Standpunkt, 3 voll., Hamburg, Felix Meiner Verlag. Tr. it., La psicologia dal punto di vista empirico, 3 voll., Roma, Laterza, 1997.

Butera, F. (1999). "L'organizzazione a rete attivata da Cooperazione, Conoscenza, Comunicazione, Comunità: il modello 4C nella Ricerca e Sviluppo", in Studi Organizzativi n²/99.

Caligiuri, M. (2002). in R.D.Steele Intelligence - Spie e segreti in un mondo aperto, Rubbettino Editore, Catanzaro.

Clemons, E., Row M. (1991). Sustaining IT advantage: The role of structural differences, MIS Quarterly, (15) (3), 275.

Cortini, M. (2008). "From corporate websites to corporate weblogs: new frontiers of organizational communication". In International Journal of Knowledge, Culture \& Change Management, 2.

Cortini, M. (2009). "New Horizons in CSP and Employee/Employer Relationship: Challenges and Risks of Corporate Weblogs", in Employ Respons Rights Journal. 21:291-303.

Cortini, M. (2005). Nuove prospettive in psicologia del marketing e della pubblicità, Guerini Scientifica, Milano.

Drucker, P.F. (1993). Gestire il futuro, Sperling \& Kupfer Editori, Milano.

Drucker, P.F. (1988). The coming of the new organization, Harvard Business Review, (66) (1), 4553.

Expert System, Cogito, in http://www.expertsystem.it.

Festinger, L. (1954). "A theory of social comparison processes", in Human Relations, 7, pp. 117-140.

Foucault, M. (1984). Histoire de la sexualitè, Gallimard, Parigi; trad. it. Storia della sessualità, Milano, Feltrinelli.

Giannuli, A. (2009). Come funzionano i servizi segreti, Adriano Salani Editore, Milano.

Goffman, E. (1981). Forms of Talk, Philadelphia: University of Pennsylvania Pres.; tra it. Forme del parlare. Il Mulino 1987.

Herring, J.P. (1996). "Creating the Intelligence system that produces analytical intelligence", in Gilad B., Herring J. (Eds.), The art and science of business intelligence (pp. 53-81). Greenwich, CT: JAI Press.

Herring, J.P. (1998). "What Is Intelligence Analysis?", in Competitive Intelligence Magazine, 1(2), 13-16.

Iacono, G. (2000). L'organizzazione basata sulla conoscenza, Franco Angeli Milano.

Intelligence and data integration, http://www.intelligrate.it/attività/ci.html.

Invernizzi, E. (1993 ). "Dalla comunicazione interna alla comunicazione organizzativa", L'impresa, n. 1.

Ivone, R. (2005). "Imprese e psicologia - Arriva in Italia l'ego-surfing", in Panorama Economy, 26 settembre.

Kozinets, R.V. (2000). "The Field Behind the Screen: Using the Method of Netnography To Research Market-Oriented Virtual Communities", in Journal of Marketing Research.

Le Bon, G. (1895). Psicologia delle folle, Alcan, Parigi.

Leidner, D.E., Elam, J.J. (1995). "The impact of executive information systems on organizational design, intelligence, and decision making", in Information Systems Research, 64564.

Lewin, K. (1951). Field theory in social science, New York, Harper \& Row.

Locke, C., Levine, R., Searls, D., Weinberger, D. (2000). The Cluetrain Manifesto: The end of business as usual, Cambridge, MA: Perseus Books. Locke, E.A. (2003). Handbook of principles of organizational behavior, Blackwell publishing.

Malfanti, F. (2005). "Il ruolo dell'analista di Intelligence", in Intelligrate, febbraio.

McGonagle, J.J, Vella, C.M. (2003). The Manager's Guide to Competitive Intelligence. Praeger, Westport, CT.

Michaeli R., (2010). "Training to become a Cl professional", in http://www.competitive-intelligence.com

Miller, S.H. (2001). "Competitive Intelligence - An overview", in Competitive Intelligence Magazine.

Morelli, M. (2003). Teorie e tecniche della comunicazione d'impresa, ETS, Pisa.

Nacci, G. (2007). "Intelligence delle fonti aperte: per una ontologia ingenua", in Intelligence \& Storia Top Secret, n. 3.

Nacci, G. (2008). "OSINT investigativa - Tecnologie ed analisi delle informazioni", in Intelligence \& Storia Top Secret, n. 8.

Pavlov, I.P. (1903) La scoperta del riflesso condizionato.

Putnam, L., Phillips N., Chapman P. (1996). Metaphors of Communication and Organization, in S.R Clegg, C. Hardy, W.R. Nord (eds.), Handbook of Organization Studies, Sage, London, pp.375-408.

Quaglino, G., Mander M. (1987). I climi organizzativi, II Mulino, Bologna.

Romano, D.F., Felicioli, R.P. (1992). Comunicazione interna e processo organizzativo, Cortina Editore, Milano.

Salas, E., Cannon-Bowers, J. A., Rhodenizer, L., and Bowers, C. A. (1999). "Training in organizations: myths, misconceptions, and mistaken assumptions", in Personnel and Human Resources Management, (17), 12361.

Shelfer, K. (2003). "Cl Education that Advances CI Practice", in Competitive Intelligence Magazine, Vol 6, n 4; July-August 2003.

Sidoti, F. (2000). Morale e metodo nell'intelligence, Cacucci Editore, Bari.

Skinner, B.F. (1948). Walden Two, 30, Hackett Publishing Co., Indianapolis.

Steele, R.D. (2002). Intelligence - Spie e segreti in un mondo aperto, a cura di Caligiuri M., Rubbettino Editore, Catanzaro.

Stephens, K.K., Dailey, S.L. (2014). "Human Resources Development in a Technology-Infused Workplace", in IGI-Global.

Stoica, M., loanid A., Dumitrescu F. (2014). "Impact of the new media knowledge on the organization management", in Network Intelligence Studies, Volume II, Issue 1(3).

Synthema, in http://www.synthema.it/index.php/Soluzioni.

Tapscott, D., Caston, C. (1993). Paradigm shift: The new promise of information technology, New York: McGraw-Hill.

Wagner, R. (2006). "Competitor Analysis: Craft, Art or Science?" Keynote DCIF Annual Conference, Dresden.

Watzlawick, P., Beavin, J.H., Jackson, D.D. (1971). Pragmatica della comunicazione umana, Astrolabio Ubaldini, Roma.

Wundt, W. (1874). Grundzüge der physiologischen Psychologie, Leipzig: Wilhelm Engelmann. 